May 1998

IFP-759-UNC

\title{
Neutron Electric Dipole Moment and Spontaneous CP Breaking.
}

\author{
Paul H. Frampton and Masayasu Harada \\ Department of Physics and Astronomy, \\ University of North Carolina, Chapel Hill, NC 27599-3255
}

\begin{abstract}
In a model where CP is spontaneously broken, the aspon model, it is shown by extending previous arguments about $\mathrm{B}$ decay and the value of $\operatorname{Re}\left(\epsilon^{\prime} / \epsilon\right)$ in the kaon system, that the neutron electric dipole moment $D_{n}$ is bounded from below by $D_{n} \geq 10^{-29}$ e.-cm.
\end{abstract}

Typeset using REVTEX 
The electric dipole moment $\mathbf{D}$ of a neutron (NEDM) must be proportional to its spin $\mathbf{S}$ and is hence odd under T or CP and under P. The energy D.E is odd under CP and even under $\mathrm{P}$ because $\mathbf{E}$ is odd under $\mathrm{P}$ and even under CP. Thus a non-vanishing NEDM would be an explicit example of $\mathrm{CP}$ violation, outside of the kaon system.

The discovery of a NEDM will be very important in formulating the theory of CP violation where the problem is the fact that only one (complex) parameter, $\epsilon_{K}$, has been accurately measured. The second parameter in the kaon system, $\operatorname{Re}\left(\epsilon^{\prime} / \epsilon\right)$, remains uncertain - it is still consistent with zero - and the CP asymmetries in B meson decays have yet to be determined. Any further experimental measurement of $\mathrm{CP}$ violation will constrain the viable theories.

The experimental limit on $D_{n}$ is presently $D_{n} \leq 10^{-25}$ e.cm. [1,2] but it is possible [3] that this limit will be pushed back by several orders of magnitude in the forseeable future, even a limit of $10^{-30}$ e.cm. being conceivable. On the other hand, the prediction of the KM mechanism within the framework of the Standard Model is $D_{n} \simeq 2 \times 10^{-32}$ e.cm. [4] which is beyond the reach of any planned experiments.

In a model of spontaneously broken CP symmetry [8,9] one may simultaneously solve the strong CP problem (without an axion) and accommodate the observed CP violation in the kaon system. In an economical model, the Standard Model is augmented by an additional $U(1)$ gauge group under which all the usual three generations of quarks and leptons are neutral, as are the established twelve gauge bosons $\left(\gamma, Z, W^{ \pm}, g^{a}\right)$ and the single Higgs doublet. One adds a non-chiral quark doublet $(U, D)$ which carries the new charge, and two complex Higgs scalar singlets $\chi^{\alpha}$. These $\chi^{\alpha}(\alpha=1,2)$ carry an equal new charge. With this arrangement, the generalized $(4 \times 4)$ quark mass matrix has a real determinant and hence at tree-level the value of $\bar{\Theta}$ is zero if the underlying theory respects CP symmetry.

Denoting the (complex) VEVs of the $\chi^{\alpha}$ by $<\chi^{\alpha}>$ and the Yukawa coupling constants to the $i$ th generation by $h_{i}^{\alpha}$ useful parameters are

$$
x_{i}=\sum_{\alpha=1}^{\alpha=2} h_{i}^{\alpha}<\chi^{\alpha}>
$$


These $x_{i}$ are complex, but for convenience we shall write $x$ to denote $x=\left|x_{i}\right|$, the modulus, and taken to be generation independent since the limits on $x$ are not sensitive to the generation considered.

In [10], it was shown that the predicted value of $\bar{\Theta}$ is estimated from a one-loop diagram as:

$$
\bar{\Theta}=\frac{\lambda x^{2}}{16 \pi^{2}}
$$

This follows from the diagram shown in Fig1. In the notation of [9] the imaginary part gives a contribution:

$$
\bar{\Theta}(u p)=\frac{1}{\sqrt{2}} \frac{1}{(4 \pi)^{2}} \sum_{\alpha=1, l=1}^{\alpha=2, l=3} h_{l}^{\alpha} \operatorname{Im}\left[x_{l}^{*}\right] \lambda_{\alpha} \frac{\kappa}{M}
$$

which gives the estimate of Eq.(2), from which it follows that $\lambda x^{2}$ is less than $\sim 10^{-8}$. Here $\lambda_{\alpha}$ is the coefficient of the quartic interaction between the two types of Higgs $|\phi|^{2}\left|\chi_{\alpha}\right|^{2}$, and $\lambda$ with no subscript is an average value. (Actually there are three independent $\lambda$ corresponding to indices $11,12+21,22$ but our estimates will not distinguish these).

The neutron electric dipole $D_{n}$ has been calculated in terms of $\bar{\Theta}$ long ago [11, 12] with the result that

$$
D_{n} \simeq 10^{-15} \bar{\Theta} e . c m \text {. }
$$

and so we know from $D_{n} \leq 10^{-25}$ e. cm. empirically that $\bar{\Theta} \leq 10^{-10}$. Here we seek to establish a lower limit on the prediction for $\bar{\Theta}$ in the present model.

Firstly, we look at the kaon system for which the parameter $\left|\epsilon_{K}\right|$ is given by [9, 13]:

$$
\left|\epsilon_{K}\right|=\frac{1}{\sqrt{2} \Delta m_{K}} m_{K} \frac{f_{K}^{2}}{3} \frac{2}{\kappa^{2}} x^{4}
$$

Using $\left(\Delta m_{K} / m_{K}\right)=7.0 \times 10^{-15}, f_{K}=0.16 \mathrm{GeV}$ gives the relationship between $x^{2}$ and the $U(1)_{\text {new }}$ breaking scale:

$$
\kappa / x^{2}=2.9 \times 10^{7} \mathrm{GeV} .
$$


Thus, if we insist that the $U(1)_{\text {new }}$ is broken above the electroweak breaking scale $(\sim$ $250 \mathrm{GeV}$ ) then:

$$
x^{2} \gtrsim 10^{-5} \text {. }
$$

From Eq.(2), this means that $\lambda<10^{-3}$.

In [10], it was further argued on the basis of naturalness that $\lambda>10^{-5}$ which (if taken at face value) would imply that $\bar{\Theta}>10^{-12}$ and hence $D_{n}>10^{-27}$ e. cm.

But here we use what is a more solid, and more conservative, bound to achieve our lower limit on $D_{n}$. Our bound follows from the fact that the $|\phi|^{2}\left|\chi_{\alpha}\right|^{2}$ interaction receives a one-loop correction from the quark loop box where three sides are the top quark and the fourth is the heavy $U$ quark (see Fig. 2). The full $\lambda$ is given by:

$$
\lambda=\lambda_{\text {tree }(\text { bare })}+\lambda_{1-\text { loop }}(\text { including counterterm })+\text { higher loops. }
$$

and the 1-loop finite contribution, for the dominant diagram (Fig 2), neglecting the quark masses and taking $h_{3}^{\alpha}, g_{t}$ as the respective Yukawa couplings to $\chi_{\alpha}$ and $\phi$ of the third generation

$$
\lambda_{1-\text { loop }} \simeq \int \frac{d^{4} k}{(2 \pi)^{4}}\left|h_{3}^{\alpha}\right|^{2}\left|g_{t}\right|^{2} \frac{1}{k^{4}}+\text { counterterm }
$$

which implies that the lowest value for $\lambda$ (without accidental cancellations) is:

$$
\lambda \gtrsim \frac{x^{2}}{16 \pi^{2}}
$$

Combining $\operatorname{Eqs}(2)$ and (10) then gives the estimate for $\bar{\Theta}$ of

$$
\bar{\Theta} \geq \frac{x^{4}}{16 \pi^{4}}
$$

which implies that $x^{2} \leq 10^{-3}$ (incidentally in full agreement with $\left[10\right.$ ) and that $10^{-10} \geq$ $\bar{\Theta} \geq 10^{-14}$.

From Eq(团), this then give our required lower limit on the neutron electric dipole moment of 


$$
D_{n} \geq 10^{-29} \text { e.cm. }
$$

This is more than two orders of magnitude greater than the prediction of the KM mechanism and thus provides yet another distinguishing feature of spontaneous CP violation. Recall that this spontaneous $\mathrm{CP}$ violation model predicts, as reflects its superweak nature, that $\operatorname{Re}\left(\epsilon^{\prime} / \epsilon\right) \simeq 10^{-5}$ [13] (compared to $O\left(10^{-3}\right)$ in the KM mechanism) and that the angles $\beta, \gamma$ of the unitarity triangle measurable in B Factories are a few milliradians [10] (rather than $O(1)$ as in the KM mechanism). It is very exciting to realize that at least one of these different $\mathrm{CP}$ breakdown mechanisms must be experimentally refuted with the advent of $\mathrm{B}$ Factories.

This work was supported in part by the US Department of energy under Grant No. DE-FG05-85ER-40219. 


\section{REFERENCES}

[1] N.F. Ramsey, Ann. Rev. Nucl. Sci. 32, 211 (1982).

[2] K.F. Smith et al., Phys. Lett. B234, 191 (1990).

[3] M.S. Freedman, G.R. Ringo and T.W. Dombeck, A Proposed Method for Measuring the Electric Dipole Moment of the Neutron Using Acceleration in an Electric-Field Gradient and Ultracold Neutron Interferometry. nucl-ex/9804003.

[4] E.P. Shabalin, Sov. J. Nucl. Phys. 31, 864 (1980).

[5] N. Deshpande, Phys. Lett. 108B, 42 (1982).

[6] I.B. Khriplovich and A.R. Zhitnitsky, Phys. Lett. 109B, 490 (1982).

[7] B. Gavela et al., Phys. Lett. 109B, 215 (1982).

[8] P.H. Frampton and T.W. Kephart, Phys. Rev. Lett. 66, 1666 (1991).

[9] P.H. Frampton and D. Ng, Phys. Rev. D43, 3034 (1991).

[10] P.H. Frampton and S.L. Glashow, Phys. Rev. D55, 1691 (1997).

[11] V. Baluni, Phys. Rev. D19, 2227 (1979).

[12] R.J. Crewther, P. Di Vecchia, G. Veneziano and E. Witten, Phys. Lett. 88B, 123 (1979).

[13] P.H. Frampton and M. Harada, UNC-Chapel Hill Report IFP-757-UNC (1998) hepph/9803410.

\section{Figure Captions.}

Fig 1. One loop diagram of which the imaginary part contributes to $\bar{\Theta}$.

Fig. 2. One loop $|\phi|^{2}|\chi|^{2}$ counterterm for the coupling $\lambda$. 


\section{FIGURES}

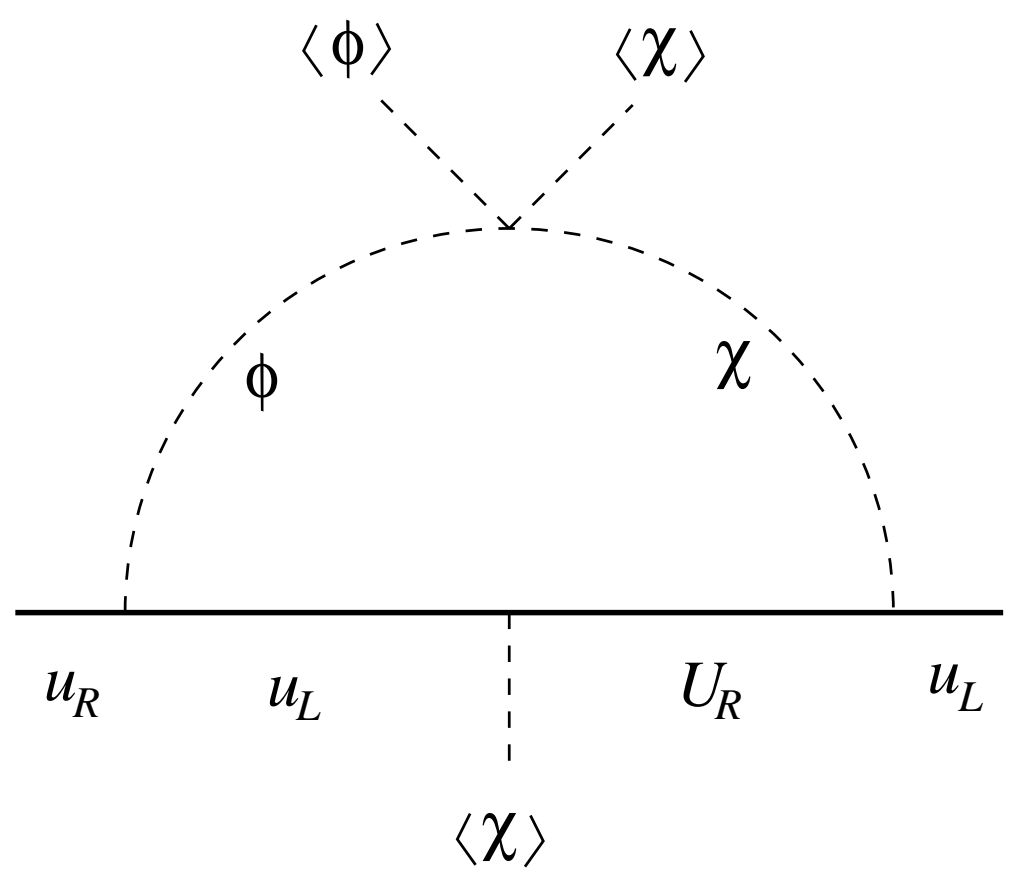

Figure 1. 


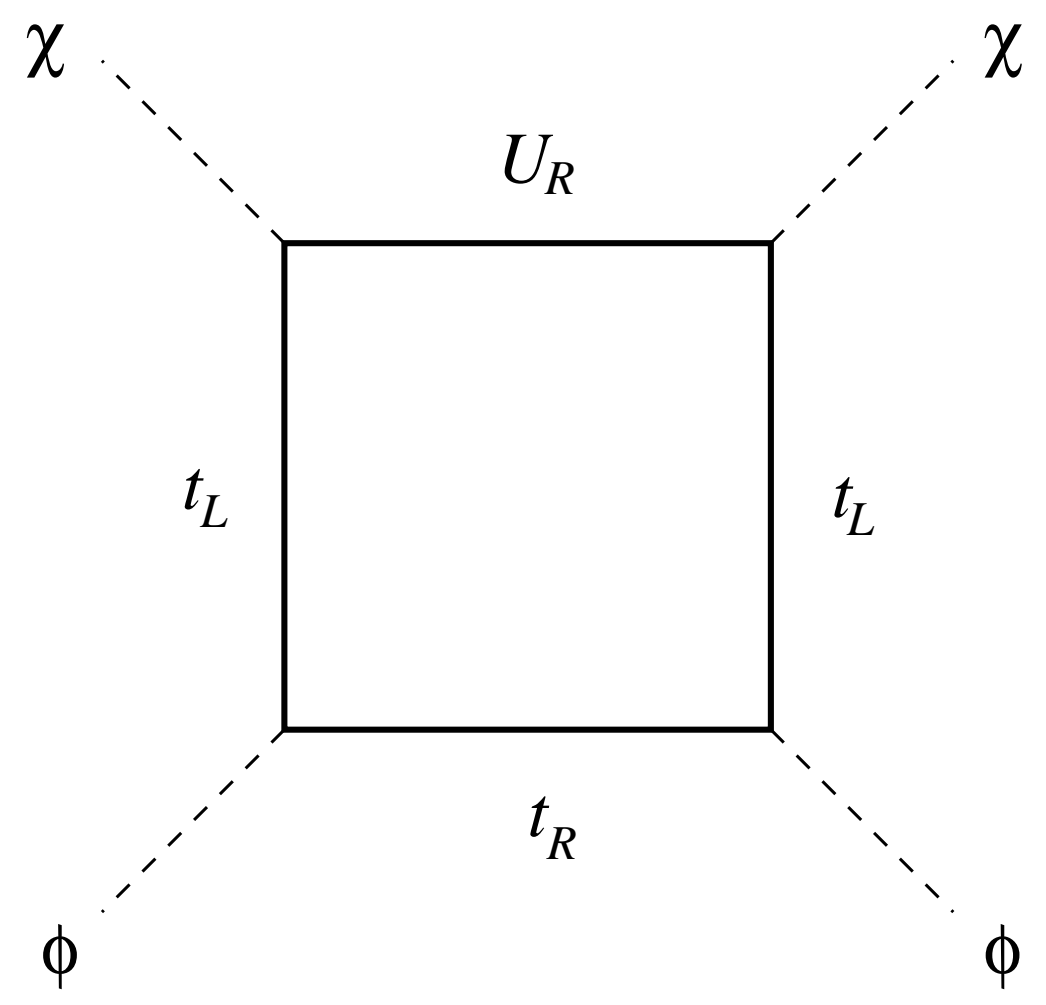

Figure 2 